\title{
Asymmetric isolated skyrmions in polar magnets with easy-plane anisotropy
}

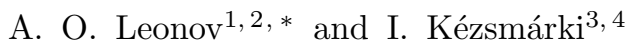 \\ ${ }^{1}$ Center for Chiral Science, Hiroshima University, Higashi-Hiroshima, Hiroshima 739-8526, Japan \\ ${ }^{2}$ Department of Chemistry, Faculty of Science, Hiroshima University \\ Kagamiyama, Higashi Hiroshima, Hiroshima 739-8526, Japan \\ ${ }^{3}$ Experimental Physics V, Center for Electronic Correlations and Magnetism, \\ University of Augsburg, Augsburg 86135, Germany \\ ${ }^{4}$ Department of Physics, Budapest University of Technology and Economics and MTA-BME \\ Lendület Magneto-Optical Spectroscopy Research Group, Budapest 1111, Hungary
}

(Dated: September 7, 2018)

\begin{abstract}
We introduce a new class of isolated magnetic skyrmions emerging within tilted ferromagnetic phases of polar magnets with easy-plane anisotropy. The asymmetric magnetic structure of these skyrmions is associated with an intricate pattern of the energy density, which exhibits positive and negative asymptotics with respect to the surrounding state with a ferromagnetic moment tilted away from the polar axis. Correspondingly, the skyrmion-skyrmion interaction has an anisotropic character and can be either attractive or repulsive depending on the relative orientation of the skyrmion pair. We investigate the stability of these novel asymmetric skyrmions against the elliptical cone state and follow their transformation into axisymmetric skyrmions, when the tilted ferromagnetic moment of the host phase is reduced. Our theory gives clear directions for experimental studies of isolated asymmetric skyrmions and their clusters embedded in tilted ferromagnetic phases.
\end{abstract}

PACS numbers: $75.30 . \mathrm{Kz}, 12.39 . \mathrm{Dc}, 75.70 .-\mathrm{i}$.

Magnetic chiral skyrmions are particle-like topological solitons with complex spin structure ${ }^{1}$ which are the solutions of the field equations of the Dzyaloshinskii's theory ${ }^{5}$. Recently, skyrmion lattice states and isolated skyrmions were discovered in bulk crystals of chiral magnets near the magnetic ordering temperatures ${ }^{6 / 8}$ and in nanostructures with confined geometries over larger temperature regions 5 12. The small size, topological protection and easy manipulation of skyrmions by electric fields and currents ${ }^{13}[15$ generated enormous interest in their applications in information storage and processing 16117 . Depending on the crystal symmetry of the host materials, distinct classes of skyrmions, such as Bloch and Néel skyrmions, or anti-skyrmions ${ }^{18}$ can be realized. In particular, Néel skyrmions were recently found in $\mathrm{GaV}_{4} \mathrm{~S}_{8}$ and $\mathrm{GaV}_{4} \mathrm{Se}_{8}$, which are magnetic semiconductors with non-chiral but polar crystal structure ${ }^{8[19}$. Néel skyrmions emerging in such multiferroic hosts are associated with an electric polarization pattern, which can be exploited for their electric field control20.

The current interest of skyrmionics is focused on isolated axisymmetric skyrmions within the polarized ferromagnetic (PFM) state of non-centrosymmetric magnets. All the spins around such skyrmions are parallel to the applied magnetic field and point opposite to the spin in the center of the skyrmion, as visualized in Figs. 1 (a) \& (c). The internal structure of such axisymmetric skyrmions, generally characterized by repulsive skyrmion-skyrmion interaction, has been thoroughly investigated theoretically ${ }^{2122}$ and experimentally by spinpolarized scanning tunneling microscopy in PdFe bilayers with surface induced Dzyaloshinskii-Moriya interactions (DMI) and strong easy-axis anisotropy 23/24. The existence region of axisymmetric skyrmions was found to be
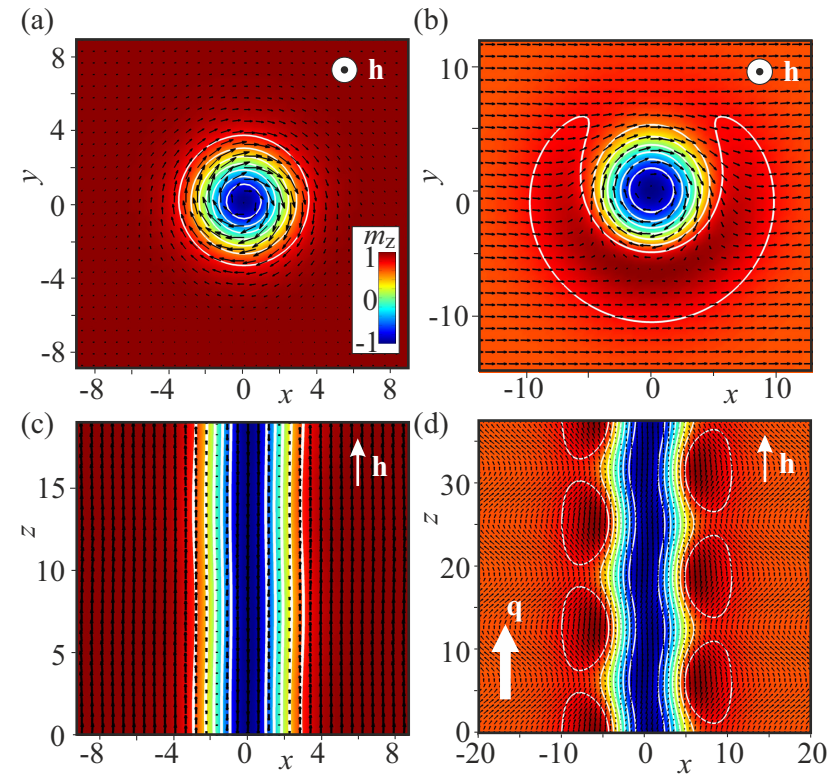

FIG. 1. (color online) Cross-sections of the internal magnetic structures of skyrmions obtained in model (1) with DMI (2) and no axial anisotropy, $k_{u}=0$. The magnetic field $\mathbf{h}$ is directed along the $z$ axis. (a) \& (c) Color plots of $m_{z}$ for an axisymmetric skyrmion embedded within the PFM state $(h$ $=0.55)$ in the $x y$ and $x z$ planes, respectively. (b) \& (d) the same for a non-axisymmetric skyrmion in the conical phase $(h$ $=0.3$, with an additional screw-like rotation alongside with the conical phase (white arrow shows the modulation vector $\mathbf{q}$ of the conical phase). The color bar in panel (a) is common for each panel. The in-plane component of the magnetization is represented by black arrows.

restricted by strip-out instabilities at low fields and a col- 
(a)

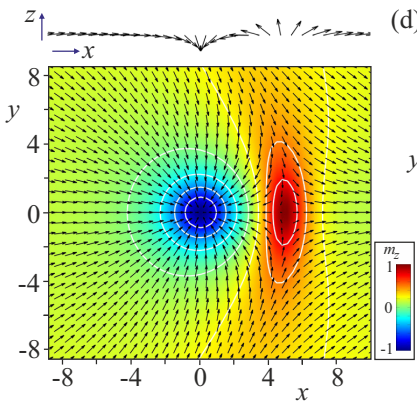

(b)

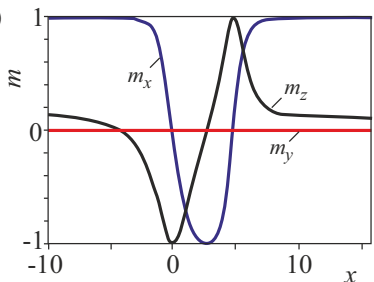

(c)

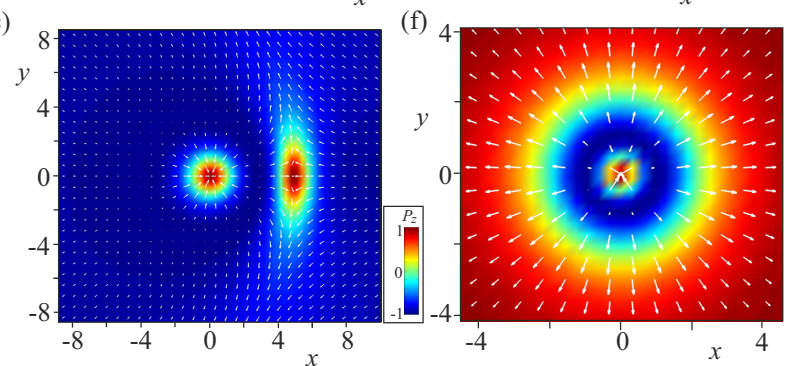

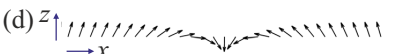

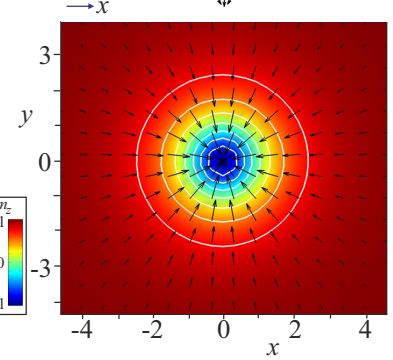

(e) 1

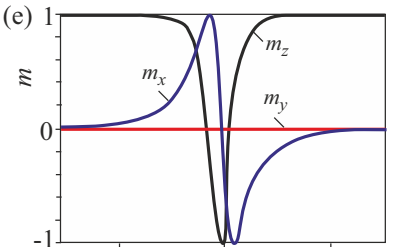

FIG. 2. (color online) Numerical solutions for skyrmions using model (1) with DMI (3). (a) - (c) Asymmetric skyrmions within the TFM state for $h=0.5$ and $k_{u}=-1.1$. (d) - (f) Axisymmetric skyrmions within the PFM state for $h=1.6$ and $k_{u}=-0.7$. The top panels are color plots of $m_{z}$ in the $x y$ plane with black arrows representing the in-plane magnetization components. The top insets show the magnetization components in the plane $x z$ across the skyrmion centers, which are additionally shown as curves in the middle panels. Note that the $m_{x}$ and $m_{z}$ components seemingly exchange roles in panels (b) \& (e), which is due to the different embedding phase, the TFM state with nearly in-plane magnetization and the PFM state with fully out-of-plane magnetization. The bottom panels are color plots of $P_{z}$ with white arrows representing the in-plane components of the electric polarization, calculated according to Eq. 6.

lapse at high fields 22 .

The internal spin pattern of isolated skyrmions can break the rotational symmetry once placed into the conical phase of bulk helimagnets ${ }^{25}$, such as the cubic B20 compounds. These skyrmions are not uniform along their axes. Though their central core region nearly preserves the axial symmetry, the domain-wall region, which connects the core with the embedding conical state, is asymmetric ${ }^{25}$. This asymmetric profile of the cross-section forms a screw-like modulation along the skyrmion core, as depicted in Figs. 1 (b) \& (d). These asymmetric isolated skyrmions, which can exhibit an attractive skyrmion-skyrmion interaction, were proposed to underlie the precursor phenomena near the ordering temper- atures in chiral B20 magnets $(\mathrm{MnSi}, \mathrm{FeGe})^{25}$ and have prospects in spintronics as an alternative to the common axisymmetric skyrmions 26 .

In this Letter we introduce a new type of isolated skyrmions within the tilted ferromagnetic (TFM) state of magnets with polar crystal structure and easy-plane anisotropy. Such skyrmions are forced to develop an asymmetric shape in order to match their spin pattern with that of the TFM state, meanwhile preserving their topological charge $q=1$. We find that-unlike the repulsive axisymmetric skyrmions and the attractive asymmetric skyrmions respectively embedded in the PFM state and the conical phase of chiral magnets - the asymmetric skyrmions emerging in the TFM state of polar magnets exhibit anisotropic inter-skyrmion potential. Depending on the relative orientation of the two individual skyrmions, this potential can be attractive, leading to the formation of biskyrmion or multiskyrmion states, or repulsive.

Chiral solitons and modulated phases can be derived by minimizing the energy functional of a noncentrosymmetric ferromagnet ${ }^{5 / 27 / 28}$ :

$$
w=\sum_{i, j}\left(\partial_{i} m_{j}\right)^{2}-k_{u} m_{z}^{2}-\mathbf{m} \cdot \mathbf{h}+w_{D} .
$$

Here, we use reduced values of the spatial variable, $\mathbf{x}=\mathbf{r} / L_{D}$ with $L_{D}=A / D$ being the periodicity of the modulated states. $A$ is the exchange stiffness constant. The sign of the Dzyaloshinskii constant $D$ determines the sense of rotation. $k_{u}=K_{u} A / D^{2}<0$ is the uniaxial anisotropy of easy-plane type, $\mathbf{m}=$ $[\sin \theta \cos \psi, \sin \theta \sin \psi, \cos \theta]$ is the unity vector along the magnetization, and $\mathbf{h}=\mathbf{H} A / D^{2}$ is the applied magnetic field in reduced units.

Depending on the crystal symmetry, the DMI energy $w_{D}$ includes certain combinations of Lifshitz invariants $\mathcal{L}_{i, j}^{(k)}=m_{i} \partial m_{j} / \partial x_{k}-m_{j} \partial m_{i} / \partial x_{k}{ }^{[215}$. Particularly, for cubic helimagnets, such as $\mathrm{MnSi}^{6}, \mathrm{FeGe} e^{10}, \mathrm{Cu}_{2} \mathrm{OSeO}_{3} 29$ and $\beta$-type Mn alloys $\$$, belonging to the chiral $23(\mathrm{~T})$ and $432(\mathrm{O})$ crystallographic classes, the DMI is reduced to the following form

$$
w_{D}=\mathbf{m} \cdot(\nabla \times \mathbf{m})
$$

and stabilizes Bloch-type modulations. Two types of isolated skyrmions have been found to exist for DMI (2): axisymmetric skyrmions within the PFM state and asymmetric skyrmions within the conical phase, respectively visualized in Fig. 1 (a) \& (b).

In the phase diagram of noncentrosymmetric ferromagnets with DMI (2) and easy-plane uniaxial anisotropy, the conical phase is the only stable modulated state 31 . Other states including skyrmion lattices and spirals are only metastable solutions of the model, though can be stabilized in real materials by several means, such as thermal fluctuations and confined geometry. Axisymmetric isolated skyrmions exist as metastable excitations of the 
(a)

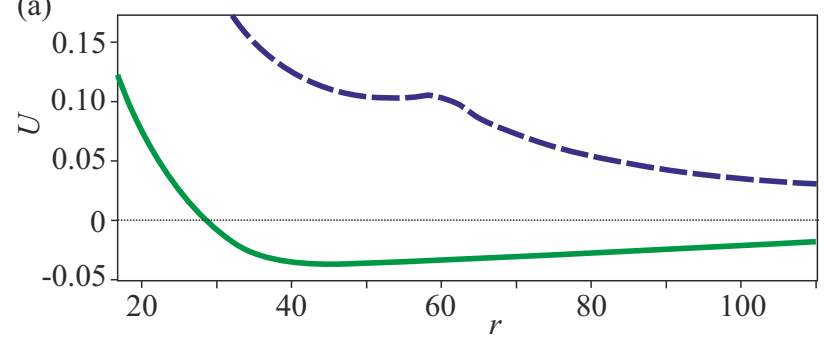

(b)

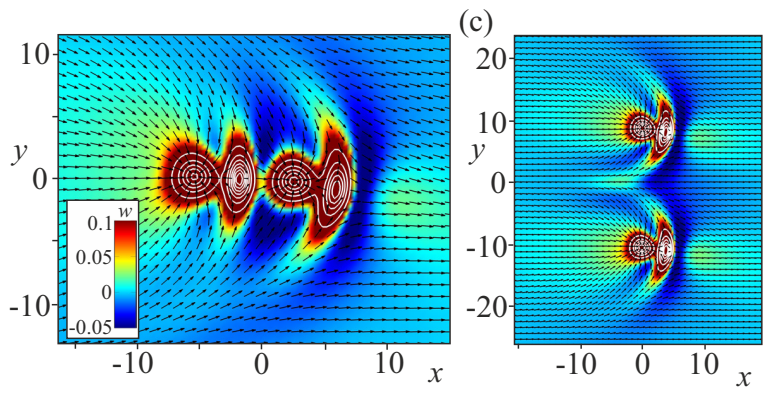

FIG. 3. (color online) (a) The inter-skyrmion potential $U$ versus the distance $r$ between the skyrmion centers. $U(r)$ was calculated by imposing the constraint, $m_{z}=-1$, at the skyrmion centers 58 and minimizing the energy with respect to spins at all other sites $\left(h=0.5, k_{u}=-1.1\right)$. Contour plots of the energy density distribution $w(x, y)$ for (b) a bound pair of asymmetric skyrmions in the head-to-head configuration and (c) a repulsive pair of asymmetric skyrmions in the side-byside configuration. Black arrows represent the in-plane magnetization components. The energy scale, common for panels (b) \& (c), is chosen in the range $(-0.05,0.1)$ to highlight the inter-skyrmion regions.

PFM state for $h>0.5-2 k_{u}$, while asymetric skyrmions are present for $h<0.5-2 k_{u} 31$.

The form of DMI is different for materials belonging to polar uniaxial $\left(\mathrm{C}_{n v}\right)$ crystallographic classes,

$$
w_{D}=m_{x} \partial_{x} m_{z}-m_{z} \partial_{x} m_{x}+m_{y} \partial_{y} m_{z}-m_{z} \partial_{y} m_{y},
$$

which are the subject of the present work. This type of DMI can stabilize skyrmions and spirals (cycloids) of Néel-type with the rotation plane of the magnetization including the wave vector and the polar axis (Fig. 2). As the conical phase is suppressed in this case, the phase diagram reveals wide regions of different states with oneand two-dimensional modulations, as discerned in Figs. 1 and 4 of the Supplement 33 . The TFM and PFM states in polar magnets with easy-plane anisotropy host two distinct types of isolated skyrmions, as shown in Fig. 2.

Isolated axisymmetric Néel skyrmions within the PFM phase are characterized by azimuthal $(\theta)$ and polar $(\psi)$ angles of the spins according to

$$
\theta=\theta(\rho), \quad \psi=\varphi .
$$

Here the boundary conditions are $\theta(0)=\pi, \theta(\infty)=0$, while $\varphi$ and $\rho$ are cylindrical coordinates of the spatial variable.
On the other hand, the isolated Néel skyrmions embedded in the TFM phase are confined by the following in-plane boundary conditions:

$$
\theta(0)=\pi, \theta(\infty)=\theta_{T F M}=\arccos \left(h / 2 k_{u}\right) .
$$

These boundary conditions violate the rotational symmetry, forcing the skyrmions to develop an asymmetric shape.

The results of the numerical minimization of the energy functional (1) with boundary conditions (5) are shown in Figs. 2 (a) \& (b). When the canted moment of the TFM state is along the $y$ axis, the asymmetry is clearly reflected in both $m_{x}$ and $m_{z}$. As implied by $m_{z}$, such skyrmions consist of a strongly localized nearly axisymmetric core and an asymmetric transitional region toward TFM state. From the left side of the depicted skyrmion, the magnetization rotates directly from $\theta_{T F M}$ to $\theta=\pi$ in the skyrmion center. In contrast, at the right side, the magnetization first passes through $\theta=0\left(m_{z}=1\right)$ and then converges back to $\theta_{T F M}$. This is the reason of the crescent-shaped anti-skyrmion-like region with a positive energy density over the TFM state, shown in Figs. 3 (b) \& (c), which is necessary to maintain the topological charge $q=1$ of such a highly distorted asymmetric skyrmion.

As already mentioned, Néel skyrmions in insulating hosts can have a polar dressing in addition to their magnetic pattern. The asymmetry of Néel skyrmions embedded in the TFM phase is also reflected in the spatial pattern of their electric polarization, as visualized in Fig. 2 (c) in comparison with the polar pattern of an axisymmetric Néel skyrmion in Fig. 22(f). The magnetically induced polarization was calculated using the lowest-order magnetoelectric terms allowed in materials with $\mathrm{C}_{4 v}$ or $\mathrm{C}_{6 v}$ symmetry:

$$
\mathbf{P}=\left[\alpha m_{x} m_{z}, \alpha m_{y} m_{z}, \beta m_{z}^{2}+\gamma\left(m_{x}^{2}+m_{y}^{2}\right)\right] .
$$

For the polarization patterns shown in Figs. 2 (c) and (f), we set $\alpha=\beta=-\gamma$ and used the normalization condition, $|\mathbf{m}|=1$. While the feasibility of electric-fielddriven switching has been demonstrated already for Néel skyrmions $\frac{15}{15}$, the asymmetry of the polarization pattern, characteristic to the isolated skyrmions studied here, can be exploited to control their orientation by in-plane electric fields.

Asymmetric skyrmions within the TFM phase can be considered as $x y$ cross-sections of asymmetric skyrmions in the conical phases, shown in Figs. 1 1 (b) \& $(d)^{25 / 26}$. However, the DMI term (3) stabilizing Néel skyrmions does not support any modulation along the $z$ axis. Thus, the asymmetry of Néel skyrmions created within the TFM state is uniform along the $z$ axis, which results in a non-trivial character of inter-skyrmion potential, displayed in Fig. 3 (a). Figs. 3 (b) \& (c) present energy density distributions in skyrmion pairs for two mutual orientations, head-to-head and side-by-side. In the 


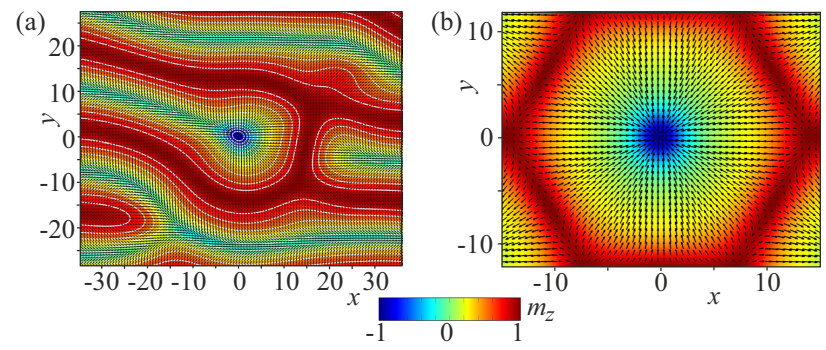

FIG. 4. (color online) (a) Instability of an isolated Néel skyrmion with respect to the elliptical cone state as obtained for $h=0.5$ and $k_{u}=-0.5$. (b) The structure of skyrmions in the hexagonal skyrmion lattice for $h=0.5$ and $k_{u}=-0.7$.

head-to-head configuration, skyrmions form pairs with a fixed inter-skyrmion distance, implying the attractive nature of their interaction, as clear from the green curve in Fig. 3 (a). Therefore, these skyrmions are expected to form 1D chains running along the canted magnetization component of the TFM phase ${ }^{36}$. The calculated inter-skyrmion potential for the side-by-side configuration, the blue curve in Fig. 3 (a), reveals the repulsive character of skyrmion-skyrmion interaction at large distances with a local minimum (or saddle point) at smaller distances. Such a behavior of the inter-skyrmion potential is related to the positive and negative asymptotics of the energy density toward the TFM state. In general, we argue that the inter-skyrmion potential inherently contains a number of minima separated by saddle points (see also Supplement for additional details).

In contrast, the asymmetric pattern of Bloch skyrmions is tightly linked to the conical modulation of the host phase and rotates around the $z$ axis in the same way for each individual skyrmions. This synchronized screw-like rotation of the asymmetry for a pair of such skyrmions leads to an overall attractive potential, by averaging over head-to-head, side-by-side and intermediate configurations alternating along the $z$ axis 25 .

The asymmetric skyrmions within the TFM state can exist only for $k_{u}<-1$, i.e. require a relatively strong easy-plane anisotropy. For $k_{u}>-1$ isolated skyrmions undergo an instability towards the elliptical cone state, as demonstrated in Fig. 5 (a) (see Ref. 33 and Supplement for a detailed information on the structure of the elliptical cone and its lability region). This instability resembles the elliptical instability of skyrmions into spirals considered in Ref. 22 and allows to generalize the considered phenomenon: isolated skyrmions tend to elongate into one-dimensional states (elliptical cones or spirals) which have smaller energy for given control parameters.

The TFM state turns into the PFM state with $\theta_{P F M}=$ 0 at the line $h=2 k_{u}$. In the PFM state the rotational symmetry is recovered and isolated skyrmions become axisymmetric with $\theta=\theta(\rho)$ and $\psi=\phi$, as shown in Figs. 2 (d) \& (e). The core region and the surrounding ring have positive and negative energy densities, respectively, implying a repulsive skyrmion-skyrmion interaction ${ }^{1}$.
Néel skyrmions can also form the thermodynamically stable skyrmion lattices. Skyrmions within unit cells of such lattices have perfectly hexagonal shape, as seen in Fig. 5 (b), and do not bear any hint on the asymmetric skyrmion structure or skyrmion instability into the elliptical cone.

Results obtained within the model (1) with DMI (3) are valid for bulk polar magnets with axial symmetry as well as for thin films with interface induced DMI. In particular, bulk polar magnetic semiconductors $\mathrm{GaV}_{4} \mathrm{~S}_{8}{ }^{8}$ and $\mathrm{GaV}_{4} \mathrm{Se}_{8} \frac{19}{19}$ with the $C_{3 v}$ symmetry possess uniaxial anisotropy of easy-axis and easy-plane type, respectively. Since the magnitude of the effective anisotropy in these lacunar spinels strongly varies with temperature, these material family provides an ideal arena for the comprehensive study of anisotropic effects on modulated magnetic states ${ }^{19}$. Skyrmions were also studied experimentally in various systems with interface induced $\mathrm{DM} \mathrm{L}^{23|24| 37 / 38}$. In these thin film structures, the rotational symmetry can also be broken by different anisotropic environments due to lattice strains or reconstructions in the magnetic surface layer ${ }^{39}$, as has been discussed recently for the double atomic layers of $\mathrm{Fe}$ on $\operatorname{Ir}(111)^{40}$. These structural anisotropies also promote the formation of asymmetric skyrmions.

In conclusion, we found a new type of isolated skyrmions emerging in tilted FM states of polar magnets with easy-plane anisotropy. These novel solitonic states are characterized by an asymmetric shape and an anisotropic inter-skyrmion potential. Our results are of particular interest for 2D materials like thin films, surfaces, interfaces, where easy-plane anisotropy can coexist with Rashba-type spin-orbit coupling, activated by the broken surface-inversion symmetry 33135 . In order to fully explore their characteristics and functionalities, the internal structure of these asymmetric skyrmions should be studied experimentally, as was done for the axisymmetric individual skyrmions within polarized FM states 23124 .

The authors are grateful to K. Inoue, A. Bogdanov, and Y. Togawa for useful discussions. This work was funded by JSPS Core-to-Core Program, Advanced Research Networks (Japan). This work was supported by the Hungarian Research Fund OTKA K 108918.

\section{SUPPLEMENTARY INFORMATION}

\section{A. Elliptical cone}

We analyze solutions of the model (1) starting from one-dimensional cycloids and elliptical cones (Fig. 5). We direct their $\mathbf{q}$-vectors along $x$, and thus get the polar and azimuthal angles as functions of only one spatial coordinate: $\theta=\theta(x), \psi=\psi(x)$. The twisting DMI acquires the following form:

$$
w_{D}=-\cos (\psi) \partial_{x} \theta+(1 / 2) \sin (2 \theta) \sin (\psi) \partial_{x} \psi
$$




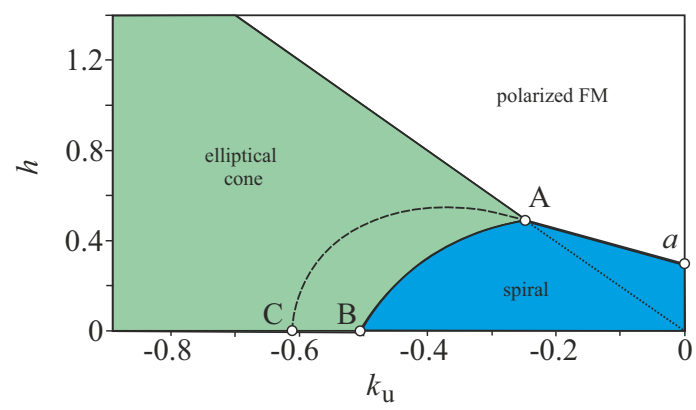

FIG. 5. (color online) (a) Magnetic phase diagram of the solutions for model (1) with easy-plane uniaxial anisotropy. Only homogeneous and one-dimensional modulated structures are taken into account. Filled areas designate the regions of thermodynamical stability of corresponding phases: blue shading - cycloidal spiral, green shading - elliptical cone, white shading - polarized ferromagnetic state. Line $A-B$ indicates the second-order phase transition between two spiral phases. At the line $a-A-C$ the cycloidal state infinitely expands into the homogeneous state, although the line $A-C$ is never reached and represents a theoretical result for cycloids with fixed boundary conditions $\theta(0)=0, \theta(p / 2)=\pi$ obtained in Ref. 31

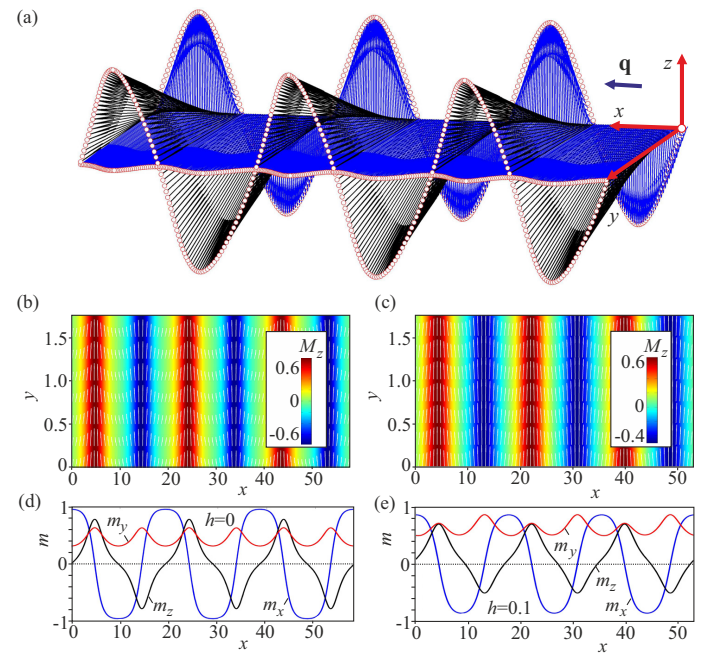

FIG. 6. (color online) (a) The structure of the elliptical cone state in chiral magnets with easy-plane anisotropy constructed for $k_{u}=-0.6, h=0$. The $\mathbf{q}$-vector is directed along $x$ axis. In the elliptic cone, the magnetization (shown as black arrows and additionally highlighted by red circles at their heads) traces out an elliptic cone, i.e., the cross section is an ellipse rather than a circle. The blue arrows show the corresponding projections on the $x z$ and $x y$ planes. The magnetization components $m_{x}(x)$ and $m_{z}(z)$ (blue arrows in the $x z$ plane) give rise to DMI (7); the in-plane $m_{x}, m_{y}$ components (blue arrows in the $x y$ plane) define the energy of the uniaxial anisotropy. (b), (c) Variations of the magnetization component $m_{z}$ along the $x$ coordinate (color plot) together with in-plane magnetization components (white arrows) are shown for two values of the field, $h=0$ and $h=0.1$, respectively. (d) and (e) Corresponding magnetization components $m_{x}, m_{y}, m_{z}$ as functions of $x$. (a)

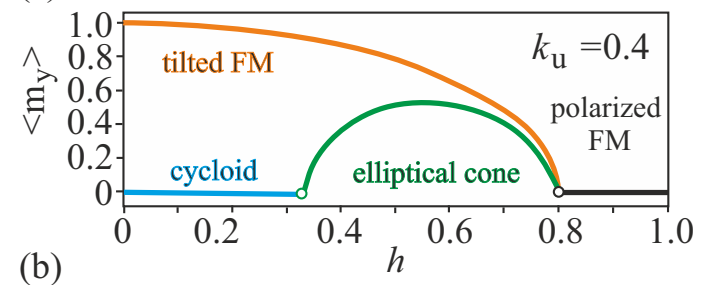

(b)

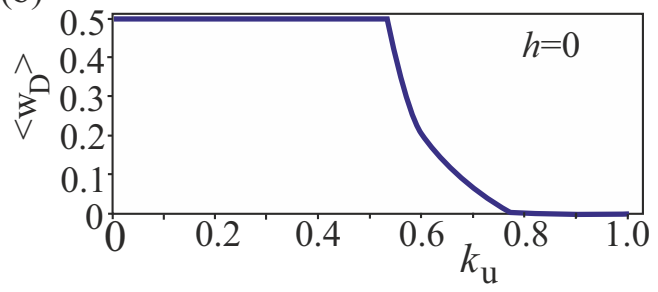

FIG. 7. (color online) (a) the field-dependence of the average $m_{y}$-component shown in the cycloidal state, elliptical cone, and the TFM state. The color markings of the curves correspond to the colors of the stability regions at the phase diagram. $m_{y}=0$ in cycloids and polarized FM state; it is non-zero in TFM and elliptical cone states. (b) The rotational DMI energy is shown in dependence on the value of $k_{u}$ for $h=0$. The DMI energy steeply goes to 0 around $k_{u}=-0.75$ although the elliptical cone as the global minimum of the system exists till $k_{u}=-1$.

For a cycloid, $\psi=0$ and thus $w_{D}=\partial_{x} \theta$ (the notation $\partial_{x}=\partial / \partial x$ is used). For $h=0$ the average value of DMI energy equals $1 / 2$ like in undistorted spirals with uniform rotation (Fig. 7 (b)). In the applied magnetic field, the rotational part of the cycloids is squeezed to narrow domain walls between wide domains polarized along the field. Thus, the rotational energy gradually decreases to 0 . Usually, such a cycloid is considered to satisfy the boundary conditions: $\theta(0)=0, \theta(p / 2)=\pi$ where $p$ is a pitch of a cycloid subject to minimization. Thus, in the case of the easy-plane uniaxial anisotropy as well as in the case of easy-axis anisotropy $\underline{32}$, the cycloid is believed to infinitely expand its period and transform into the TFM state at the line $a-A-C$ (Fig. 5 see also Fig. 12 in Ref. 31).

In our simulations, however, we show that the cycloidal spiral rather gives rise to the elliptical cone (Fig. 6) at the line $A-B$ (Fig. 5). An elliptical cone (EC) was introduced in Ref. 33, Spins in this phase trace out a cone with an elliptical cross-section (Fig. 6 (a) - (e)). The angle of this cone is defined by the corresponding angle of the tilted FM state, $\theta=\arccos \left(h / 2 k_{u}\right)$. Such an EC develops from a cycloidal state by the secondorder phase transition and gradually increases its $m_{y^{-}}$ component (Fig. 7 (a)). At the line $h=2 k_{u}$, EC continuously transforms into the polarized FM state (Fig. 5). For $k_{u}<-1$ the EC transforms into the tilted FM state. We note that the TFM state is isotropic in plane. On the contrary, the EC develops its in-plane component perpendicular to the q-vector. By this, the unidirectional sense of the magnetization rotation in EC is 


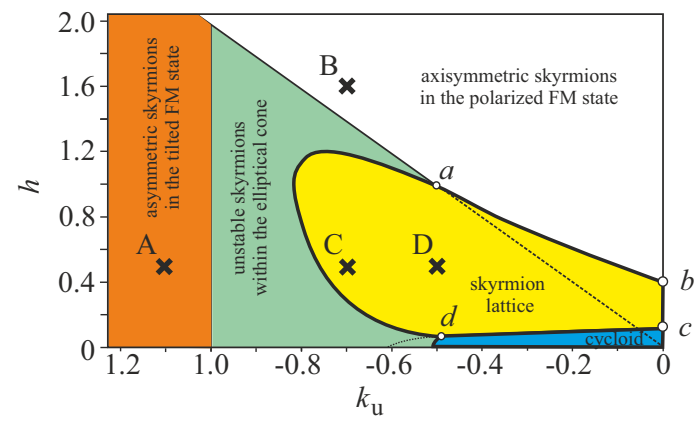

FIG. 8. (color online) Magnetic phase diagram of the solutions for model (1) with easy-plane uniaxial anisotropy and DMI (3). Filled areas designate the regions of thermodynamical stability of corresponding phases: blue shading - cycloidal spiral, green shading - elliptical cone, white shading - polarized ferromagnetic state, yellow shading - hexagonal skyrmion lattice, orange shading - TFM state. Thick black lines indicate the first-order phase transitions between corresponding phases, thin black lines - the second-order phase transitions.

preserved, and the DMI energy of EC (7) is modified by the additional $\psi(x)$-dependence. At the lines $k_{u}=-1$ and $h=2 k_{u}$ the rotational energy (7) falls to 0 (Fig. 7 (b)).

\section{B. The phase diagram of states}

The complete phase diagram (Fig. 8 ) of states of the model (1) has been reproduced from Ref. 33 and includes stability regions of modulated phases and regions of metastable skyrmions. Points A-D indicate parameters for different solutions used in the manuscript: point
A - asymmetric skyrmions within TFM phase (Fig. 2 (a) - (c)); point B - axisymmetric skyrmions within PFM phase (Fig. 2 (d) - (f)); point C - hexagonal skyrmion lattice (Fig. 4 (b)), point D - instability of asymmetric skyrmions with respect to the elliptical cone (Fig. 4 (a)).

The phase diagrams also allows to generalize the processes of skyrmion lattice formation. Along the line $a-b$ the skyrmion lattice appears as a result of condensation of isolated skyrmions (building blocks of the hexagonal skyrmion lattice), as found for axisymmetric skyrmions in the easy-axis case $e^{1 / 22 / 32}$. Along the line $c-d$, hexagonal skyrmion lattice may appear as a result of local cutting of the cycloid (in this sense, two merons may be considered as nuclei of the skyrmion lattic $e^{\sqrt{42}}$ ). Along the firstorder phase transition line $a-d$, however, none of the aforementioned mechanisms is appropriate. Presumably, domains of the skyrmion lattice and the elliptical cone state coexist with non-trivial domain boundary between them.

\section{Inter-skyrmion potential}

To calculate the skyrmion-skyrmion interaction potential, the following procedure was proved to be appropriate for axisymmetric skyrmions ${ }^{41}$ and asymmetric skyrmions within the conical phase ${ }^{25}$ : the energy density (1) is minimized with the constraint $m_{z}=-1$ imposed at the centers of two skyrmions as a function of the distance between skyrmion centers. This procedure, however, faces the following difficulty when applied to asymmetric skyrmions within TFM states: skyrmions will locally deform the surrounding TFM state to achieve the minimum of the system. Thus, only positions of (local and global) minima can be found precisely. To reconstruct the interaction potential with all underlying details, one should impose a control over the in-plane component of the TFM state, i.e. violate its in-plane isotropy. *leonov@hiroshima-u.ac.jp

1 A. Bogdanov and A. Hubert, J. Magn. Magn. Mater. 138, 255 (1994); 195, 182 (1999).

2 A. N. Bogdanov and D. A. Yablonskii, Zh. Eksp. Teor. Fiz. 95, 178 (1989) [Sov. Phys. JETP 68, 101 (1989)].

3 N. Nagaosa and Y. Tokura, Nat. Nanotechnol. 8, 899911 (2013).

4 U. K. Rößler, A. A. Leonov, and A. N. Bogdanov, J. Phys. Conf. Ser. 303, 012105 (2011).

${ }^{5}$ I. E. Dzyaloshinskii, Sov. Phys. JETP 19, 960 (1964).

6 S. Mühlbauer, et al., Science 323, 915919 (2009).

7 H. Wilhelm, M. Baenitz, M. Schmidt, U. K. Roessler, A. A. Leonov, and A. N. Bogdanov, Phys. Rev. Lett. 107, 127203 (2011).

8 I. Kezsmarki et al., Nat. Mater. 14, 11161122 (2015).

9 X. Z. Yu, Y. Onose, N. Kanazawa, J. H. Park, J. H. Han, Y. Matsui, N. Nagaosa, and Y. Tokura, Nature (London) 465, 901 (2010).

10 X. Z. Yu et al., Nat. Mater. 10, 106109 (2011).

11 H. Du et al., Nat. Commun. 6, 7637 (2015).
12 D. Liang, J. P. DeGrave, M. J. Stolt, Y. Tokura, and S.Jin, Nat. Commun. 6, 8217 (2015).

13 T. Schulz et al., Nat. Phys. 8, 301304 (2012).

14 F. Jonietz, S. Mühlbauer, C. Pfleiderer, A. Neubauer, W. Mnzer, A. Bauer, T. Adams, R. Georgii, P. Böni, R. A. Duine, K. Everschor, M. Garst, A. Rosch, Science 330, 1648 (2010).

15 P-J. Hsu et al., Nat. Nanotechnol. 12 123-126 (2017).

16 J. Sampaio, V. Cros, S. Rohart, A. Thiaville, and A. Fert, Nat. Nanotechnol. 8, 839844 (2013).

17 E. M. R. Tomasello, R. Zivieri, L. Torres, M. Carpentieri, and G. Finocchio, Sci. Rep. 4, 6784 (2014).

18 A. K. Nayak, V. Kumar, P. Werner, E. Pippel, R. Sahoo, F. Damay, U. K. Rößler, C. Felser, S. S. P. Parkin, arxiv. 1703.01017.

19 S. Bordacs et al., [arXiv: link available soon]

20 E. Ruff et al., Sci. Advances 1, e1500916 (2015).

21 A. Bogdanov, A. Hubert, phys. stat. sol. (b) 186, 527 (1994). 
22 A. O. Leonov, T. L. Monchesky, N. Romming, A. Kubetzka, A. N. Bogdanov, and R. Wiesendanger, New J. of Phys. 18, 065003 (2016).

23 N. Romming, C. Hanneken, M. Menzel, J. E. Bickel, B. Wolter, K. von Bergmann, A. Kubetzka, and R. Wiesendanger, Science 341, 636 (2013).

24 N. Romming, A. Kubetzka, C. Hanneken, K. von Bergmann, and R. Wiesendanger, Phys. Rev. Lett. 114, 177203 (2015).

25 A. O. Leonov, T. L. Monchesky, J. C. Loudon, and A. N. Bogdanov, J. Phys.: Condens. Matter. 28, 35LT01 (2016).

26 A. O. Leonov, J. C. Loudon, A. N. Bogdanov, Appl. Phys. Lett. 109, 172404 (2016).

27 P. Bak and M. H. Jensen, J. Phys. C: Solid State Phys. 13, L881 (1980); O. Nakanishi, A. Yanase, A. Hasegawa, and M. Kataoka, Solid State Comm. 35, 995 (1980).

28 A. A. Leonov, Ph.D. thesis, Technical University Dresden (2012).

29 S. Seki, X. Z. Yu, S. Ishiwata, and Y. Tokura, Science 336, 198-201 (2012).

30 Y. Tokunaga et al., Nat. Commun. 6, 7638 (2015).

31 M. N. Wilson, A. B. Butenko, A. N. Bogdanov, and T. L. Monchesky, Phys. Rev. B 89094411 (2014).

32 A. B. Butenko, A. A. Leonov, U. K. Rößler, and A. N. Bogdanov, Phys. Rev. B 82, 052403 (2010).
33 J. Rowland, S. Banerjee, M. Randeria, Phys. Rev. B 93, 020404 (2016).

${ }^{34}$ U. Güngördü, R. Nepal, O. Tretiakov, K. Belashchenko, A. A. Kovalev, Phys. Rev. B 93, 064428 (2016).

35 S. Banerjee, J. Rowland, O. Erten, M. Randeria, Phys. Rev. X 4, 031045 (2014).

36 S.-Z. Lin, A. Saxena, C. Batista, Phys. Rev. B 91, 224407 (2015).

37 B. Dúpe, G. Bihlmayer, M. Böttcher, S. Blügel, and S. Heinze, Nat. Commun. 7, 11779 (2016).

38 S. Woo, K. Litzius, B. Krüger, M. Im, L. Caretta, K. Richter, M. Mann, A. Krone, R. Reeve, M. Weigand et al., Nat. Mater. 15, 501 (2016).

39 J. Hagemeister, E. Y. Vedmedenko, and R. Wiesendanger, Phys. Rev. B 94, 104434 (2016).

40 P. J. Hsu, A. Finco, L. Schmidt, A. Kubetzka, K. von Bergmann, and R. Wiesendanger, Phys. Rev. Lett. 116, 017201 (2016).

41 A. O. Leonov and M. Mostovoy, Nat. Commun. 6, 8275 (2015).

42 M. Ezawa, Phys. Rev. B 83, 100408 (2011). 